\title{
Pamela H. Smith, Amy R. Meyers et Harold J. Cook (dir.), Ways of making and knowing. The material culture of empirical knowledge
}

Anne Arbor, The University of Michigan Press, 2014

\section{Marie Thébaud-Sorger}

\section{OpenEdition}

\section{Journals}

\section{Édition électronique}

URL : http://journals.openedition.org/artefact/744

DOI : 10.4000/artefact.744

ISSN : 2606-9245

Éditeur:

Association Artefact. Techniques histoire et sciences humaines, Presses universitaires du Midi

\section{Édition imprimée}

Date de publication : 15 juin 2017

Pagination : 235-239

ISBN : 978-2-7535-6525-8

ISSN : 2273-0753

\section{Référence électronique}

Marie Thébaud-Sorger, «Pamela H. Smith, Amy R. Meyers et Harold J. Cook (dir.), Ways of making and knowing. The material culture of empirical knowledge », Artefact [En ligne], 5 | 2016, mis en ligne le 15 novembre 2017, consulté le 24 septembre 2020. URL : http://journals.openedition.org/artefact/744 ; DOI : https://doi.org/10.4000/artefact.744

Ce document a été généré automatiquement le 24 septembre 2020.

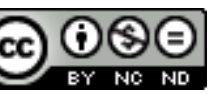

Artefact, Techniques, histoire et sciences humaines est mise à disposition selon les termes de la Licence Creative Commons Attribution - Pas d'Utilisation Commerciale - Pas de Modification 4.0 International. 


\section{Pamela H. Smith, Amy R. Meyers et Harold J. Cook (dir.), Ways of making and knowing. The material culture of empirical knowledge}

Anne Arbor, The University of Michigan Press, 2014

Marie Thébaud-Sorger

\section{RÉFÉRENCE}

Pamela H. Smith, Amy R. Meyers et Harold J. Cook (dir.), Ways of making and knowing. The material culture of empirical knowledge, Anne Arbor, The University of Michigan Press, $2014,430 \mathrm{p}$.

1 Fruit d'une collaboration à la croisée de l'histoire de l'art, de l'artisanat et de la médecine, l'ouvrage dirigé par Pamela H. Smith, Amy Meyers et Harold Cook part du postulat fort et stimulant de saisir la constitution des savoirs sur la nature par la prise en compte de la pluralité des relations entre "faire» et "connaître». En faisant résonner l'histoire des savoir-faire, des pratiques médicales et commerciales et des pratiques de collections, les éditeurs ont intégré également la réflexion menée avec les conservateurs, restaurateurs et artisans spécialisés dont l'ouvrage marque à la fois un aboutissement et une étape.

Dans leur introduction, les auteurs questionnent l'héritage intellectuel qui a départagé le monde des idées de celui des pratiques, faisant un bref retour historiographique sur ce qu'a apporté l'approche matérielle des sciences dans le renouvellement de la compréhension des relations entre sciences et arts - retour succinct et limité principalement aux apports anglo-saxons. Cette approche n'est pas nouvelle, mais elle s'est très largement consolidée et imposée, notamment à travers les travaux précédents de Pamela Smith, et dont témoignent également d'autres entreprises collectives 
d'envergure comme l'opus dirigé par Lissa Roberts, Simon Shaffer et Peter Dear, The mindful hand ${ }^{1}$. Ces chemins, largement empruntés depuis deux décennies $-\mathrm{y}$ compris par la longue lignée qui de Ann Blair à Françoise Wacquet récemment ${ }^{2}$ démontre que l'histoire intellectuelle ne peut faire abstraction des conditions proprement matérielles qui président à l'élaboration de connaissances -, ont conduit à réintégrer la matérialité des gestes et les dispositifs techniques dans l'élaboration du savoir, à remettre en cause les frontières qui séparent l'activité théorique des activités pratiques et à examiner la nature de ces relations. En explicitant le rôle majeur des savoirs empiriques, du «faire» comme moyen de "connaître ", William Eamon avait également montré naguère que les arts de faire sous-tendant la culture des secrets et des recettes à la Renaissance avaient largement anticipé des pratiques expérimentales savantes aux fondements de l'émergence et des transformations de la science moderne ${ }^{3}$. Si replacer les savoirs de la main au cœur de l'élaboration de la connaissance opère un déplacement épistémique majeur, permettant d'appréhender par les processus de fabrication, l'expérimentation, l'observation et la généralisation de la nature (p.13), des approches renouvelées se sont fait également jour sur la manière dont la production matérielle est aussi forgée par des visions du monde et des formes d'intellections spécifiques, d'intelligence technique comme a pu le montrer récemment Liliane Hilaire-Pérez".

3 Reprenant dans leur propos liminaire la figure de la déesse Athéna pour s'intéresser à la mètis, cette notion complexe que Détienne et Vernant définissaient comme « ruses, astuces, stratagème ", les auteurs soulignent pour leur part que, si Athéna apparaît comme la déesse de l'intelligence et de la stratégie guerrière, c'est bien grâce à la mètis, à la forme d'intelligence pratique au cœur de la création et de la transformation des matériaux, que celle-ci a pu s'armer de casque et de bouclier. Situer alors la culture matérielle, les objets et leur mode de production au cœur de l'ouvrage permet d'aborder l'histoire des sciences non comme une histoire des concepts, mais bien comme une histoire de faire et utiliser les artefacts. La ruse d'Athéna sert ici de guide pour penser le savoir empirique qui se déploie à travers les objets, opérant un double mouvement qui examine tant les conditions matérielles du travail intellectuel que les intellections propres aux productions matérielles. Ces processus sont examinés ici par le prisme des savoirs de la nature entendus ici au sens large (chimique, botanique, physiologique, etc.). En sortant de l'espace savant stricto sensu des laboratoires afin d'embrasser d'autres sites, ateliers, collections, boutiques, tribunaux, l'ouvrage met en évidence la variété des pratiques et la pluralité des acteurs qui contribuent à la production des savoirs. Construit autour de quatorze études de cas et d'un épilogue, il met en scène le travail des artisans, marchands, collectionneurs, naturalistes, de praticiens divers en Europe et en Amérique du nord entre le $\mathrm{xvI}^{\mathrm{e}}$ et le $\mathrm{xIX}$ siècle et se déploie autour de trois thèmes généraux qui se recouvrent et construisent la chronologie de l'ouvrage.

4 Premièrement, une série de contributions touchent au travail de la matière, reliant art de faire et pratiques artisanales, dans différents contextes de productions et d'usages. Le bel essai de Pamela Smith ouvre le bal et, à partir de la métallurgie au $\mathrm{XVI}^{\mathrm{e}}$ siècle, envisage la pratique artisanale comme philosophie naturelle. À travers l'analyse des ingrédients utilisés par des artisans, elle met en lumière la cosmologie singulière qui habite les choix des praticiens dans leur travail. Susann Butters revisite les notions d'art, d'ingenium, alliant compétences et invention sur la matière en se concentrant sur 
la pratique et les arts visuels de l'Italie de la Renaissance. Alicia Weisberg-Roberts s'attache à la production de la teinture noire pour les textiles au xvII e siècle, notamment à partir du logwood (bois de campèche) initiant des réseaux d'échanges et de circulations entre la curiosité d'un physicien amateur, Henry Bargham, et le naturaliste et collectionneur Hans Sloane. Alicia Rankins, quant à elle, montre que l'élaboration des savoirs médicaux passe par des savoir-faire techniques car la fabrication de remèdes nécessite de construire des dispositifs de distillation dont elle étudie l'appropriation par les élites (l'impératrice du Saint-Empire et la princesse du Portugal) à travers leur mise en pratique du traité de 1512 de Hieronymus Brunshwig. Insistant eux aussi sur l'importance des sens dans une pratique répétée d'expérimentations, Patrice Wallis et Catherine Wright investissent les formes d'expertise des substances produites par des artisans londoniens au XVII ${ }^{\mathrm{e}}$ siècle au sein des guildes et notamment celle des apothicaires.

5 Un deuxième ensemble s'attache globalement aux techniques requises par les savoirs botaniques, notamment dans le contexte des échanges transatlantiques au XVIII ${ }^{\mathrm{e}}$ siècle. Mark Laird et Karen Bridgman étudient les techniques de transport et de culture des plantes, tandis que Joel T. Fryre trace le commerce de graines mené par John Bartram entre Philadelphie et l'Europe. Lisa Ford examine le travail de retraduction des savoirs arboricoles mené au XIX ${ }^{e}$ siècle des Michaux père et fils également entre la France et les États-Unis, et notamment le traité de ce dernier, François André Michaux, Histoire des arbres forestiers de l'Amérique septentrionale. Elisabeth Yale enfin analyse les listes de requêtes établies pour faciliter les échanges et la collaboration avec les observateurs et naturalistes britanniques au XVII ${ }^{\mathrm{e}}$ siècle.

On repère ici à l'œuvre des formes de classement et de catégorisation résultant de ces échanges commerciaux ou savants et qui ouvrent sur un troisième ensemble de textes portant sur le rôle des artefacts matériels dans la production savante, les considérant comme de véritables outils de pensée. De manière convaincante, l'ouvrage s'attache de manière neuve aux effets cognitifs induits par ces techniques de conservations, comme la production spécifique de meubles de rangements à compartiments qu'étudie Glenn Adamson, pour l'organisation et la conservation des collections dans les cabinets de curiosité et qui donne lieu à un travail d'ébénisterie bien particulier; ou encore la mise au point des techniques de préservation des spécimens et surtout de conservation anatomique des cadavres mises au point par le hollandais Louis de Bils dont Harold $\mathrm{J}$. Cook démontre le rôle majeur dans l'émergence de nouveaux savoirs sur le corps à la période moderne ; ces savoir-faire sont mis en écho avec les pratiques contemporaines menées dans les musées et qui tentent de lutter contre la fragilité et la désagrégation des spécimens conservés, abordées par Mary M. Brooks. Ce dernier volet insiste en outre sur l'importance des artefacts dans le développement théorique de l'approche du vivant. Ainsi, la constitution de figures dessinées engendre un travail formel basé sur l'analogie et la comparaison permettant de construire des relations entre les objets. Sachiko Kusukawa met en lumière l'impact de la production artisanale d'orfèvrerie de Wenzel Jamnitzer (également étudié par P. Smith ${ }^{5}$ ) et sa technique de moulage sur le vivant, sur la nouvelle manière que développe, au milieu du XvI ${ }^{\mathrm{e}}$ siècle, Conrad Gessner de saisir et produire des images "d'après nature", ad vivum, tandis qu'Horst Bredekamp explore la manière dont le travail théorique de Darwin s'esquisse à travers des artefacts matériels et dessinés qui constituent un chemin dans la mise en ordre de 
sa pensée : l'étude de la structure du corail et la figuration du jeu de ses ramifications propose un premier modèle analogique pour penser les processus de l'évolution.

De manière habile, des problématiques transversales resserrent donc l'ouvrage. En premier lieu celle des savoirs des artisans et de leurs relations à l'élite des savoirs, à travers des sources variées, comptes, expertises juridiques, imprimés commerciaux. La frontière est poreuse entre artiste et artisan ou entre l'artisan et le chirurgien au $\mathrm{XVI}^{\mathrm{e}}$ siècle, comme l'évoque A. Rankin avec le cas de Brunshwig : le travail technique est au cœur de la fabrication de dispositifs permettant la production des savoirs médicaux (remèdes), définie alors comme science (Wissenschaft). On voit en dehors des espaces institutionnels, notamment celui de l'université et des académies, d'habiles artisans, marchands, amateurs gentilshommes, contribuer largement à créer des outils de savoirs dans le plein sens du terme. Comme l'écrit H. Cook, Louis de Bils est aussi un véritable entrepreneur (vente de cadavres embaumés, animation d'un théâtre d'anatomie et cours public payant) qui tente de monnayer son secret, stimulant de ce fait la recherche alternative par de multiples expérimentations et imitations, décloisonnant en tous les cas l'espace de la production savante pour l'inscrire dans un espace social beaucoup plus large. Il démontre toutefois que, si ces savoirs résultent des nouvelles possibilités techniques, les chirurgiens et médecins reprennent in fine la main et renforcent l'autorité médicale de l'Université avec des outils qui avaient été conçus initialement dans une intention autre par le monde artisanal et commercial. Ces pratiques artisanales en action apparaissent également dans l'enquête de Patrick Wallis et Catherine Wright. L'étude de l'administration des expertises permet de cerner une activité quotidienne où sont mobilisées des méthodes empiriques et sensitives extrêmement fiables, seules à même de juger de la qualité d'un produit. On le voit, la question économique et marchande constitue un facteur majeur où s'opère la stabilisation de corps de connaissances précises sur la nature des substances et leurs usages. L'essor de nouvelles consommations au cours des XVIII ${ }^{\mathrm{e}}$ et $\mathrm{XIX}^{\mathrm{e}}$ siècles produit de nouvelles taxonomies, dans le cas, par exemple, du commerce des graines, des plants (nursery) ou des demandes consuméristes pour divers objets.

8 Par ailleurs la production imprimée, traités, planches et gravures, constitue également un des fils rouges du livre. Elle est examinée sous deux angles, d'une part, la relation entre l'esquisse, le dessin, le relevé, le questionnaire, l'herbier et la production imprimée (dans le cas des ouvrages de Gessnerou de Michaux où l'on voit à l'œuvre la manière dont le savant supervise la production de planches gravées) et, d'autre part, dans les dynamiques de circulation et d'appropriation des savoirs qu'ils rassemblent, mettent en ordre et diffusent. Si, toutefois, la spécificité de l'écrit technique, à la fois prescriptif et théorique, n'est pas véritablement interrogée en tant que tel, comme ont pu l'engager toute une série de travaux depuis les recherches d'Hélène Vérin sur les réductions en art $^{6}$, la variété des cas présentés témoigne non seulement de l'élargissement des publics de la littérature technique, mais aussi de l'hétérogénéité de ces supports : traités populaires en langue vernaculaire ou latine, éditions luxueuses, relations de voyage jusqu'aux imprimés commerciaux comme les catalogues de vente. De ce fait, la place des savoirs empiriques et leur formalisation peuvent être perçus dans des contextes de production et d'usage très différents, de la lecture des remèdes par une élite sise dans les cours de la Renaissance à la diffusion auprès d'un large lectorat porté par les nouveaux marchés de l'économie coloniale. 
9 Ainsi, tout en démontrant qu'une histoire intellectuelle des sciences de la nature ne peut être entreprise sans être articulée à une série d'opérations matérielles, l'ouvrage permet d'examiner ces interrelations. En traitant chaque sujet en vertu de son rapport à la production de la science, l'ouvrage engage moins symétriquement l'analyse avec les formes d'intelligence artisanale et technique qui pourtant sont présentes dans bien des cas présentés, cas qui se limitent plutôt à une frange de l'élite, producteurs et consommateurs, scrutés autour d'objets souvent exceptionnels. Mais son grand intérêt réside dans le fait de placer l'artefact en position centrale. Plaçant au même niveau en quelque sorte les productions de l'art, les supports papier aux formats les plus divers, la chaussure conservée au V\&A, les planches d'échantillons de tissu, les herbiers et meubles de rangement : comme le souligne à juste titre Malcom Baker dans sa postface, ces spécimens constituent des archives à part entière à partir desquelles on peut renouveler les enquêtes - l'exemple de la Kunstschrank (1632) de Philipp Hainhofer, à Augsburg, qu'évoque Glenn Adamson en offre l'illustration aboutie: son examen permet de renverser l'approche du cabinet de curiosités comme « microcosme » et d'en déceler une autre logique, morphologique, celle qui incarne la lutte pragmatique non pour exhiber des merveilles, mais pour être en mesure de tout contenir, ranger, subdiviser, voire dérober au regard parfois dans des tiroirs et des cases, les éléments naturels et artificiels (pièces, fleurs séchées, textiles, pierres) inscrits dans l'aménagement de l'espace domestique du XVII ${ }^{e}$ siècle.

Emblématique du material turn, l'ouvrage pose un regard original sur les savoirs par le bais de la culture matérielle et témoigne des renouvellements féconds qu'il génère, suscitant initiatives de recherches et de pédagogies - allant de l'étude des collections aux processus de reconstitutions - nées du dialogue fructueux entre recherches académiques et institutions muséales. Par ces solides études de cas, toutes utiles, dont on doit souligner que la diversité ne diminue en rien la belle homogénéité, l'ouvrage coordonné par Smith, Meyers et Cook trace le chemin d'une analyse qui se revendique justement plurielle, visant à explorer la diversité des manières dont on peut faire et connaître, s'articulent et font émerger, de la production des arts à la celle de la science, un ensemble de savoirs dépassant les approches disciplinaires. Ce faisant, il nous convie à relire les relations et les interactions entre mondes savants et mondes de la pratique et à interroger l'épistémologie propre aux artisans ou marchands botanistes, naturalistes, médecins, sans coïncidence souvent, mais dont les différences recouvrent la construction conjointe d'un nouvel ordre des savoirs sur la nature.

\section{NOTES}

1. Peter DeAR, Lissa ROBERTS, Simon SCHAFFER (dir.), The mindful hand: inquiry and invention from the Late Renaissance to Early Industrialisation, Amsterdam, Chicago, Edita, University of Chicago Press, 2007.

2. Françoise WAQUET, L'ordre matériel du savoir. Comment les savants travaillent, $\mathrm{XVI}^{e}$-XXI ${ }^{e}$ siècle, Paris, CNRS Éditions (CNRS Philosophie), 2015. 
3. William EAMON, Science and the secrets of Nature: books of secrets in Medieval and Early Modern Culture, Princeton, Princeton University Press, 1994.

4. Liliane HILAIRE-PÉREZ, La pièce et le geste. Artisans, marchands et savoir technique à Londres au XVIII siècle, Paris, Albin Michel, 2013.

5. Pamela H. S MITH, "In a sixteenth-century goldsmith's workshop», in P. D EAR, L. ROBERTS, S. SCHAFFER (dir.), The mindful hand, op. cit., p. 33-57.

6. Hélène VéRIn et Pascal Dubourg-GLATIGNy (dir.), Réduire en art, la technologie de la Renaissance aux Lumières, Paris, MSH, 2008.

\section{AUTEURS}

\section{MARIE THÉBAUD-SORGER}

CNRS-Centre Alexandre-Koyré 